\title{
About the Surface Hardening of Tool Steels by Electrical Discharge Treatment in Electrolyte
}

\author{
Dimitar Krastev ${ }^{1, a}$, Boyan Yordanov ${ }^{1, b}$ \\ Department of Physical Metallurgy and Heat Equipment, University of Chemical Technology and \\ Metallurgy $8 \mathrm{Kl}$. Ohridski Blvd, 1756 Sofia, Bulgaria ${ }^{1}$ \\ krastev@uctm.edu ${ }^{a}$, bobsarcho@yahoo.com ${ }^{b}$
}

Keywords: electrical discharge treatment, high speed steel, microstructure

\begin{abstract}
In this study are given some results about the influence of electrical discharge treatment in electrolyte on the microstructure of high speed steel HS 6-5-2. The metallic surface after electrical discharge treatment in electrolyte has a different structure in comparison with the metal matrix, which determines different properties. It is observed remarkable increasing of hardness, strength and corrosion resistance related to the nonequilibrium phase transformations and the obtained finecrystalline microstructure.
\end{abstract}

\section{Introduction}

The metallic surface modification by electrical discharge treatment such as Electrical Discharge Machining (EDM) is of great scientific interest at last years. Normally used for manufacturing dies/moulds and aerospace components, many researchers are adapting the technique to enable surface hardening of workpieces to create, in some cases, new harder alloys on the surface of components to increase their working life and wear resistance. Such a method is the electrical discharge treatment in electrolyte, where the modification goes by a high energy thermal process in a very small volume on the metallic surface, involving melting, vaporisation, activation and alloying in electrical discharges and after that cooling of this surface with high rate in the electrolyte. The high energy process put together with the nonequilibrium phase transformations in the metallic system causes considerable modifications of the metallic surface and obtaining of layers with finecrystalline and nanocrystalline structure [1-4]. The investigations show, that obtained on tools layers have higher hardness, wear resistance and tribocorrosion resistance, which give better workability, considerable increasing of working life and wide opportunities for industrial application.

\section{Experimental}

For the experimental work is developed a laboratory installation (Fig. 1), giving opportunities for treatment of cylindrical workpieces with diameter up to $20 \mathrm{~mm}$. The electrolyte 3 is in active movement by mixing from a magnetic stirrer 4. After the passing of electric current with definite characteristics through the electrolyte between the workpiece 1 and electrode 2 starts an active sparking on the specimen surface. The sparking characteristics depend from different factors such as parameters of the electric current, type and composition of the electrolyte, movement of the workpiece and electrolyte.

The workpieces are made from high speed steel HS 6-5-2 with structure after the typical heat treatment for tools of this steel and hardness about of HV 950. The choice of high alloyed steel is founded on the opportunity for higher effectiveness of the treatment on the structure and properties of the modified surfaces after the nonequilibrium phase transformations from liquid state. 


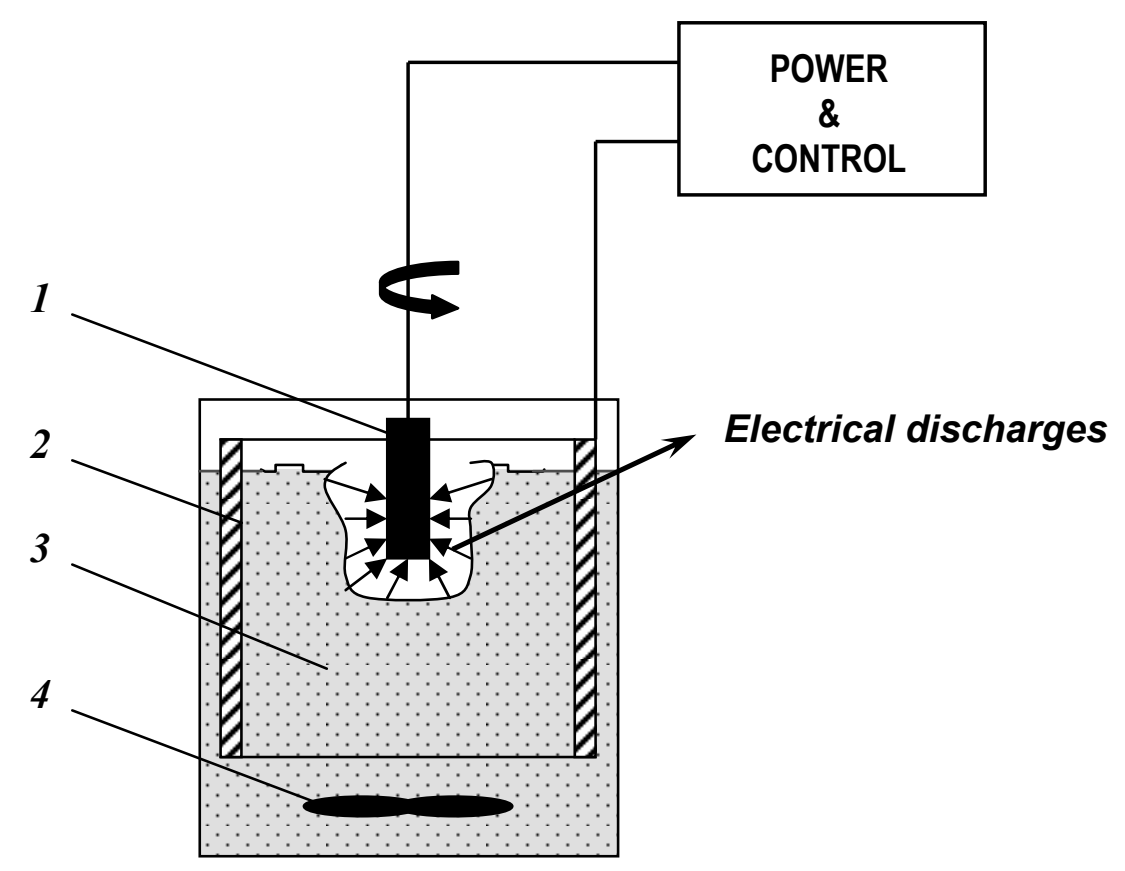

Fig. 1. Installation for electrical discharge treatment in electrolyte: 1 -workpiece, 2 -electrode, 3 -electrolyte, 4 - magnetic stirrer

\section{Results and Discussion}

In this investigation were obtained layers on the workpiece surface with approximately equal thickness, depending of the electrical current characteristics and time of treatment. The modified surfaces can be observed as a light layers on the workpieces. On Fig. 2 and Fig. 3 are shown the light microscopy microstructures of workpiece surfaces, modified for two minutes at $80 \mathrm{~V}$ and $100 \mathrm{~V}$. The thickness of the layers is $0.01 \mathrm{~mm}$ and $0.02-0.03 \mathrm{~mm}$ respectively.

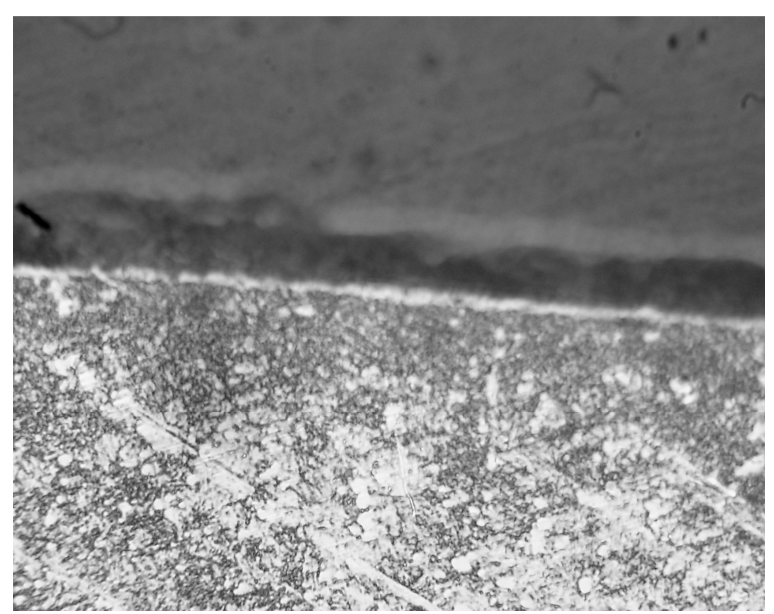

Fig. 2. Microstructure of layer, obtained for 2 min at $80 \mathrm{~V}, 600 \mathrm{x}$

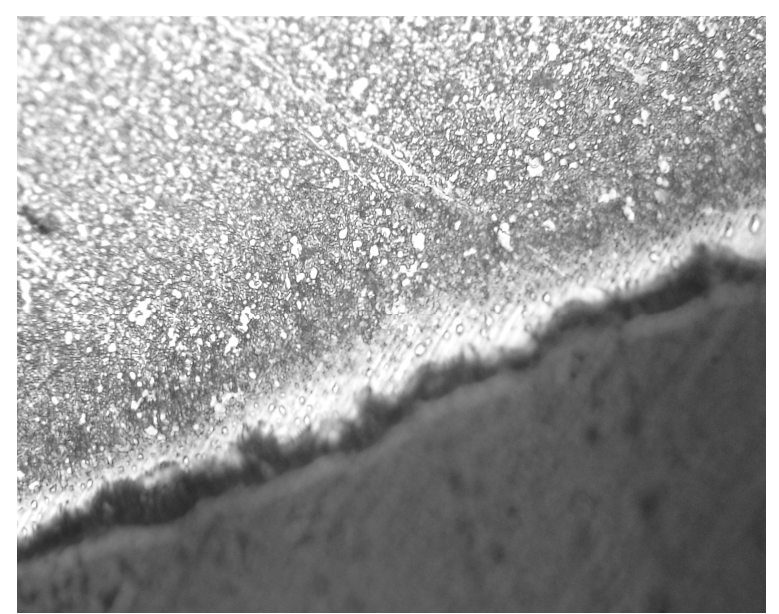

Fig. 3. Microstructure of layer, obtained for $2 \min$ at $100 \mathrm{~V}, 800 \mathrm{x}$ 
The electric discharges generate an enormous amount of heat, causing local melting on the workpiece surface and thereupon it is rapidly quenched from the liquid state by the electrolyte. This recast area has a specific structure, which is composed of several microscopic metallurgical layers, depending of machining conditions. The high rate of the recasting process gives opportunities for formation of metastable phases and considerable decreasing of grain size. The electrolyte type is of great importance for the chemical composition, microstructure and properties of the modified layer. It is on water basis and contains boron or silicon compounds. The melted and resolidified layer during this process is also referred as the "white zone", since generally no etching takes place in these areas at the metallographic preparation. This high corrosion resistance is one of the important characteristics of nanosized structures. The fine structures of modified layers with specific etching are shown after SEM investigation on Fig. 4. After modification on the workpiece surface can be observed two specific zones:

- "White zone"

- "Phase transformations zone"

The "Phase transformations zone" has different structures depending on the temperature and cooling rate. When the temperature has risen above the melting point and cooling rate is lower a zone with dendritic structure is formed - Fig. 4a. In the other case (Fig. 4b), when the temperature is in the austenitic region and the cooling rate is higher than the critical martensite is formed.

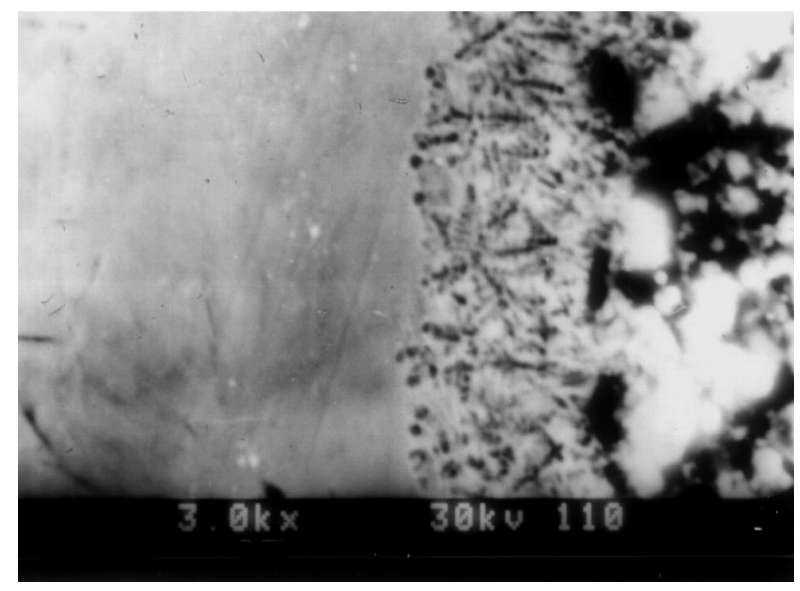

$\boldsymbol{a}$

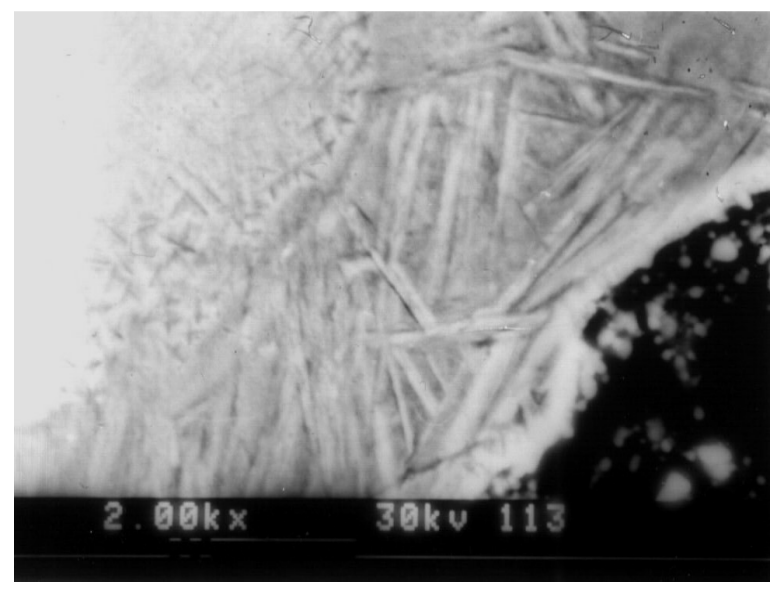

$b$

Fig. 4. SEM images of different microstructures of the modified layers on HS 6-5-2 steel surface: $a$-dendritic microstructure in the phase transformations zone, $b$-martensitic microstructure in the phase transformations zone

The hardness of the modified layers can vary considerably and depend of the treatment conditions, electrolyte composition and microstructure, but in principle it is higher then the hardness of the typical microstructure of this steel. The microhardness of the modified layers is measured by Hanneman test and shows values after the different treatments up to HV 1600, which are very higher than the microhardness of HS 6-5-2 steel microstructures after the typical heat treatment. The experiments show that tools with such surface hardness have higher wear resistance and working capacity. 


\section{Conclusions}

The electrical discharge treatment in electrolyte of high speed tool steel HS 6-5-2 causes modification of the surface and obtaining of layers with specific microstructure, higher hardness and wear resistance. The microhardness of the modified layers can vary considerably and depends of the treatment conditions, electrolyte composition and microstructure and in some cases reaches values up to $16000 \mathrm{MPa}$. Tools of this type steels with such surface hardness have higher wear resistance and working capacity.

\section{References}

[1] Gavze A. L., Matevosyan A. P., Nesterovich A. V., Bogdanovich B. Y., Metals Science and Heat Treatment, 43(2001), p. 363.

[2] Rebelo J. C., Morao Dias A., Kremer D., Lebrun J. L., Journal of Materials Processing Technology. 84(1998), p. 90.

[3] Wendl F., Wupper K., Härterei-technische Mitteilungen. 44(1989), p. 197.

[4] Amorim F.L., W.L. Weingaertner, J. of the Braz. Soc. of Mech. Sci. and Eng, 2 (2004), p. 137. 